\title{
Longitudinal Evaluation of Outcomes for Youth with Serious Emotional Disturbance during Two Years of Children's Psychosocial Rehabilitation
}

\author{
Nathaniel J. Williams \\ Michael E. Sherr
}

\begin{abstract}
This study assessed the course, rate, and significance of change in participants' day-to-day functioning during two years of Children's Psychosocial Rehabilitation (CPSR). Hierarchical linear mixed models were used to analyze Child and Adolescent Functional Assessment Scale (CAFAS) outcome data for 49 youth with serious emotional disturbance, aged 7 to 17 years. The authors estimated participants' change trajectory, difference in initial versus 16-month status, and difference in rate of change between the first 12 and last 8 months of the study. Controlling for age, participants improved by 13.73 points on the CAFAS every four months, generating a statistically and clinically significant improvement from intake to 16 months. The rate of change decreased significantly to 1.37 points per wave during the last 8 months of the study. CPSR participants improve significantly during treatment, with the majority of changes occurring in the first year.
\end{abstract}

Keywords: Serious emotional disturbance; children's psychosocial rehabilitation; community-based treatment; Child and Adolescent Functional Assessment Scale

Influenced by the national direction of treatment for youth with serious emotional disturbance (SED; Duchnowski, Kutash, \& Friedman, 2002; Ringeisen \& Hoagwood, 2002; Stroul \& Friedman, 1986), Children's Psychosocial Rehabilitation (CPSR), a Medicaid-funded, home- and community-based treatment for youth with SED, quietly sprung up in the state of Idaho in the mid-1990's and has grown into a \$38 million-a-year enterprise (Idaho Department of Health and Welfare, 2007; Williams, in press). CPSR bears many hallmarks of a quality SED-specific treatment and has shown promise empirically (Williams, in press). Despite its widespread use in Idaho, however, CPSR is understudied, with only one uncontrolled investigation of outcomes (Williams, in press). In order to determine whether or not CPSR is effective, and whether other states should adopt it, additional and more rigorous research is needed. This study advanced the evidence base on CPSR by using a retrospective longitudinal design to estimate participants' rate of change in functioning during 24 months of treatment, and differences in the rate of change between the first 12 and last 8 months of the study.

\section{Context for Children's Psychosocial Rehabilitation}

Within the Idaho Medicaid system, private, for-profit providers deliver CPSR services under a managed-care arrangement. Privatization, combined with broadly written rules which govern the program, resulted in a proliferation of providers and CPSR treatment models, all with their own treatment philosophies, staff training and supervision practices, models of intervention, and most likely, differential outcomes.

Nathaniel J. Williams, LCSW, is Children's Psychosocial Rehabilitation Program Director, CenterPointe, Inc., Nampa, Idaho. Michael E. Sherr, Ph.D., LCSW, is an assistant professor in the School of Social Work at Baylor University.

Copyright (C) 2008 Advances in Social Work Vol. 9 No. 2 (Fall 2008), 126-141 
Unfortunately, despite mandates in the Idaho Administrative Code directing the Idaho Department of Health and Welfare to evaluate the effectiveness of CPSR (Idaho Administrative Code, Dept. of Health and Welfare, Code number 16.03.09.701 16.03.10.199, 2006), no statewide efforts have been implemented to date to assess the outcomes of this innovative but expensive program.

In the absence of a statewide evaluation or any literature on CPSR, staff at one large clinic in southwestern Idaho initiated a program of research to assess the efficacy of their specific CPSR program model (Williams, in press). In an open trial design (e.g. Piacentini, Bergman, Jacobs, McCracken, \& Kretchman, 2002; Vernberg, Jacobs, Nyre, Puddy, \& Roberts, 2004), Williams (in press) compared participants' intake scores on the Child and Adolescent Functional Assessment Scale (CAFAS; Hodges, 2000a) or the Preschool and Early Childhood Functional Assessment Scale (PECFAS; Hodges, 1994) to their most recent CAFAS/ PECFAS scores across an average treatment time of 13 months. Findings were positive-78\% of participants evinced clinically significant change, defined by a drop of 20 points or more on the CAFAS or PECFAS, with a large effect size of 1.29 on CAFAS/ PECFAS total score, and significant improvements on all but the Substance Use subscales.

Although the study was an important first step in evaluating CPSR, methodological issues limited the inferences that could be drawn. First, the trial was uncontrolled, prohibiting causal inferences as to CPSR's efficacy (Kazdin, 2003). Second, the length of treatment varied widely between participants ( $\min =4$ months, $\max =36$ months), muddying the interpretation of mean changes in functioning. Third, the study lacked longitudinal data points. Pre-post evaluation models have been criticized in the literature (Bereiter, 1963; Linn \& Slinde, 1977), with experts now calling for longitudinal analyses to provide more valid estimates of changes in symptoms, functioning, or other outcomes of interest (Rogosa, Brandt, \& Zimowski, 1982; Singer \& Willett, 2003; Willett, 1989). Finally, the study was of limited duration compared to the average length of CPSR treatment; the mean treatment time was 13 months $(S D=8.89)$ with nearly half the sample receiving 8 months of treatment or less. Conversely, Williams (in press) reported that most participants remain in the program for 18 months. Further evaluation was therefore necessary to improve our understanding of CPSR's effectiveness over time and the nature of participants' change during treatment.

The current study took several logical steps forward in empirically evaluating CPSR. First, we focused our evaluation on the same CPSR program Williams (in press) examined in the original trial. Second, we employed a retrospective longitudinal design that redressed several shortcoming of the earlier report. Treatment time was held constant, easing interpretation of mean changes in functioning, and was long-term, aligning more closely with practice realities. The longitudinal design allowed us to assess the course, rate, and significance of participants' changes in functioning over time through the use of hierarchical linear mixed models (Singer \& Willett, 2003). Third, we sought to inform utilization management decisions by comparing children's intake to 12-month change trajectory to their 16- to 24-month change trajectory. One of the crucial practice questions in an open-ended service like CPSR is, "How long should treatment last?" To date, no empirical literature has addressed this issue. We therefore sought to inform the question 
of service duration by looking for differences in children's change trajectories during different periods of treatment. Taken together, these features produced a better understanding of participants' changes as they moved through the program and provided useful information for evaluation, treatment planning, and policy.

\section{Research question and hypotheses}

In sum, the current study asked "What was the course, rate, and significance of change in children's day-to-day functioning during two years of CPSR as measured by the CAFAS (Hodges, 2000a)?” We tested three hypotheses. The first related to participants' rate of change during CPSR: participants' functioning will improve significantly during their participation in the program. The second focused on the course of change: participants' functioning will improve rapidly during the first 12 months of treatment and slowly during the subsequent 8 months of treatment. Our third hypothesis related to the difference in participants' level of functioning at two points in time: after 16 and 24 months of CPSR, participants' day-to-day functioning will be significantly improved over their initial status.

\section{METHOD}

\section{Design}

This study used a retrospective 7-wave panel design diagrammed as $\mathrm{O}_{1} \times \mathrm{O}_{2} \times \mathrm{O}_{3} \mathrm{x}$ $\mathrm{O}_{4} \times \mathrm{O}_{5} \mathrm{x} \mathrm{O}_{6} \times \mathrm{O}_{7}(\mathrm{x}=$ CPSR). Outcome observations (CAFAS ratings) were conducted by CPSR treatment staff during the course of treatment; specifically, at intake and every four months thereafter. These data were subsequently gathered from clients' medical charts by the researchers. The study period spanned 24 months; this timeframe was deemed sufficient to capture significant trends and counter-trends in participants' functioning during CPSR. In order to inform questions of service duration (i.e. "How long should CPSR treatment last?”) we compared children's change trajectory during the first 12 months of the study $\left(\mathrm{O}_{1}, \mathrm{O}_{2}, \mathrm{O}_{3}, \mathrm{O}_{4}\right)$ to the trajectory during the last 8 months of the study $\left(\mathrm{O}_{5}, \mathrm{O}_{6}, \mathrm{O}_{7}\right)$. The comparison periods were chosen after visual inspection of children's change trajectories suggested that functioning leveled off significantly beyond the 12-month mark.

\section{Participants}

Participants were 49 clinic-referred children, aged 7 to 17 years $(M=11.5, S D=2.9$, $\min =7, \max =17$ ), who participated in CPSR. Inclusion criteria limited the study to CPSR participants aged 7 years or older, with two years or more of treatment. The sample included all youth who met inclusion criteria at the study site as of October 2007. Participants were diagnosed with SED, as defined by (a) one or more psychiatric diagnoses according to the most recent edition of the Diagnostic and Statistical Manual of Mental Disorders ( $4^{\text {th }}$ ed. Text Revision, American Psychiatric Association, 2000), (b) a total CAFAS score of 80 or higher, and (c) a 20 on any one of three CAFAS subscales: Moods/ Emotions, Self-harm, or Thinking. The most common primary psychiatric diagnoses were any type of Attention Deficit/ Hyperactivity Disorder (31\%) or anxiety 
disorder (31\%), including Posttraumatic Stress Disorder. Mood and depressive disorders were also common (14\% and $12 \%$, respectively). The mean number of diagnoses in the sample was $1.61(S D=.67, \min =1, \max =3)$. Diagnoses came from community practitioners who saw the children during the course of routine clinical practice in a community mental health clinic.

The sample was predominantly Caucasian (82\%) and male (63\%). The racial diversity of the sample was limited (10\% Hispanic, 8\% “Other”), reflecting a lack of diversity in the surrounding geographic area (U.S. Census Bureau, 2008). A large majority of participants lived with their biological families (84\%); $12 \%$ lived in foster care, and 4\% were adopted. All participants' families had low annual incomes which met guidelines to qualify for Idaho Medicaid.

\section{Study site}

The study took place at a large, for-profit, children's mental health clinic that specializes in the treatment of youth with SED. Located in southwestern Idaho, the clinic primarily serves Canyon County (estimated 2006 population $=173,302$; U. S. Census Bureau, 2008), one of the more populous regions in a largely rural state. Clinic referrals came from pediatricians, schools, juvenile justice, child welfare, and word-of-mouth. The majority of participants served at the clinic meet income guidelines to qualify for Idaho Medicaid.

In order to ensure the protection of human subjects, we obtained ethical review, oversight, and approval for the study from the Administrative Oversight Committee at the clinic where the study took place. Because the data were archival, stored in a retrieval system without identifiers, and not originally intended for research purposes, the risk to participants was minimal.

\section{Children's Psychosocial Rehabilitation}

CPSR programs differ considerably across the State of Idaho and beyond. Idaho State guidelines require that CPSR providers undergo a credentialing process in which their records are audited to ensure appropriate documentation of services and compliance with Idaho Administrative Code (Idaho Administrative Code, Dept. of Health and Welfare, Code number 16.03.09.701 - 16.03.10.199, 2006). In addition, all CPSR services must be prior authorized by regional mental health authorities, which ensure that clients meet enrollment criteria for CPSR and that proposed CPSR service plans comply with code.

The practice parameters outlined in Idaho Administrative Code (2006) define minimum standards for program quality and practice; they do not operationally define CPSR interventions or program features in great detail. As an example, the Code specifies minimum educational requirements for CPSR Specialists (bachelor's degree or higher in behavioral science, education or medicine), but does not outline specific preservice training requirements. The code requires CPSR Specialists to be supervised weekly, but does not dictate the format or amount of supervision they should receive. Because of this, CPSR programs vary considerably in terms of quality, staffing, training 
practices, and outcomes. The following description applies only to the CPSR program evaluated in this study.

Children's Psychosocial Rehabilitation is a home- and community-based program for youth with SED who need more intensive treatment than weekly outpatient psychotherapy, but who do not need to be psychiatrically hospitalized or placed in residential treatment. The goals of the program are to prevent youth from moving into more restrictive levels of care, to minimize the impact of mental illness, and to maximize their positive developmental trajectory. The focus is on reducing the impact of functional impairments associated with symptoms of SED. Treatment is here-and-now focused, ecologically valid (occurs in the child's home and community), and emphasizes building a positive alliance with the client and caregiver, teaching and building skills, and behavior modification. Within CPSR, psychiatric symptoms and functional impairments are thought to arise from combinations of biological, psychological, and environmental factors unique to each child and family. Similarly, each child and family is viewed as possessing unique strengths and skills that aid in remedying the presenting problems.

Each child who participates in CPSR undergoes a thorough clinical assessment that covers nine areas: psychiatric/ substance use, medical, educational, financial, social, family, housing, basic living skills, and community. The child's functioning is assessed in each area and specific strengths and weaknesses are identified. The focus is on how the psychiatric symptoms impact functioning in each area. Based on the assessment, a CPSR service plan is developed that describes (a) the child and family's broad goals, (b) concrete, measurable objectives that serve as benchmarks toward the goals, and (c) specific tasks that the CPSR Specialist, client, and family will do to achieve the objectives and goals. Tasks describe specific intervention strategies that will be used. For example, a task might state "The PSR staff will teach, practice, and review with the client skills for redirecting his anger when upset.” The PSR worker then tailors the specific teaching, practicing, and reviewing activities to the interests, developmental level, and needs of the specific client. Interventions are cognitive and behavioral in nature, and occur in relevant community settings, enhancing their ecological validity. Ideally, CPSR specialists deliver interventions within the context of a strong therapeutic alliance with the child and family; workers are intentional about fostering such alliances.

A typical CPSR service plan includes 4 to 8 hours of face-to-face skill-building intervention time per week, with an additional 2 hours per week for "collateral contacts" with important adults in the child's life (e.g. parents, teachers, coaches, youth pastor, extended family). During skill-building sessions, the CPSR Specialist works one-on-one with the child, in the context of his or her natural ecology, to teach and rehearse skills. Skill-building sessions can, and often do, include the child's parents when skill deficits involve functioning in the home. During collateral contacts, the CPSR Specialist receives information and updates from important adults in the child's life, provides education and intervention strategies to the adults, and coordinates interventions across settings. In cases where children receive additional clinical services (e.g. psychotherapy) efforts are made to coordinate intervention approaches and treatment targets. Anecdotally, CPSR treatment tends to last from 4 to 36 months, with an average of 18 to 24 months. 
CPSR Specialists are the primary intervention agents in CPSR. These individuals possess a bachelor's degree in social work, psychology, or a human services related field with 21 semester credits or more of coursework on human behavior. CPSR Specialists receive 10 hours of pre-service training, including didactic and written instruction, roleplays, and opportunities to "shadow" more experienced CPSR Specialists. Specialists receive weekly one-on-one supervision with a master's-level clinician in which they staff cases and receive guidance on clinical aspects of the work; typically this lasts from 15 to 45 minutes per week. Finally, Specialists receive 20 hours per year of continuing education related to the field of children's mental health. Standardized training materials and a manual are being developed for the CPSR program under study.

Fidelity to the intervention was not quantified in this study as no rating scales exist. Instead, as a community-based study, we relied on the clinical judgment and guidance of CPSR supervisors to ensure that treatment was provided in accordance with program standards.

\section{Outcome measures}

Child and Adolescent Functional Assessment Scale (CAFAS). The CAFAS is a clinician-administered paper-and-pencil measure designed to assess the day-to-day functioning and psychological symptoms of children ages 7- to 18-years-old (Hodges, 2000a). The CAFAS includes eight subscales which correspond to various areas of functioning or psychological well-being and include: School, Home, Community (primarily assesses delinquent acts), Behavior toward others, Moods/ emotions, Substance use, Self-harm, and Thinking. Children receive a score on each subscale ranging from 0 (minimal to no impairment) to 30 (severe impairment); subscale scores are summed to generate a total CAFAS score which can range from 0 to 240. Guidelines published on the CAFAS indicate that total scores of 50 or higher indicate the need for additional services beyond traditional outpatient care.

The CAFAS is in wide use across the United States; several states, including Idaho, use it to determine eligibility for services and to monitor outcomes in public systems of care (Bates, 2001). The psychometric characteristics of the CAFAS have been thoroughly evaluated and it has been found to have good inter-rater reliability in different samples of raters, as well as construct, concurrent, and predictive validity (Hodges, Doucette-Gates, \& Liao, 1999; Hodges \& Wong, 1996; 1997; Hodges, Wong, \& Latessa, 1998).

CAFAS ratings for this study were completed by bachelor's and master's-level clinicians during the course of CPSR treatment. Ratings reflected the child's worst level of functioning during the preceding three months. CAFAS scoring guidelines encourage raters to gather as much information about the client as possible in order to make the most accurate rating (Hodges, 2000b); accordingly, CAFAS ratings were based on parent- and child-report and on information from collateral contacts, including the child's CPSR Specialist once treatment was initiated. All CAFAS raters had successfully completed the CAFAS inter-rater reliability training and passed the inter-rater reliability test to be considered reliable CAFAS raters (Hodges, 2000b). 


\section{Analyses}

We performed univariate, indicator, and multivariate analyses on the data. Univariate analyses assessed the significance of changes in functioning from intake to each subsequent wave and from wave to wave, using a series of dependent $t$ tests. Because these were preliminary analyses we did not adjust our probability values. Indicator analyses revealed the number of participants who achieved clinically significant change from intake to 24 months. Hodges, Xue, and Wotring (2004) defined a change of 20 points or more on the CAFAS as a marker of clinically meaningful improvement; they note this corresponds to half a standard deviation on the CAFAS and a medium effect size according to Cohen's criteria (1988). Taken together, the univariate and indicator analyses provided a rough picture of the pattern and significance of participants' pre-topost, and inter-wave changes.

Our main analysis sharpened the picture using hierarchical linear mixed models (a.k.a. random coefficients regression or the multi-level model for change; Singer \&Willett, 2003). The hierarchical linear mixed models provided estimates of participants' initial status (intercept), and slope of change over time, including differences in slope between the first 12 and last 8 months of the study. Hierarchical linear mixed models allow participants' individual growth parameters (intercept and slope) to vary, an important feature in clinical work where all participants will not respond the same to an intervention (Gibbons et al., 1993; Gibbons, Hedeker, \& Davis, 1987). Mixed models also allow participants' individual growth parameters to represent a random population sample of all possible growth parameters (Singer \& Willett, 2003), thereby permitting more accurate and generalizable estimates of changes in participants' functioning. Prior to our analyses, we centered age on its mean (11.5 years), forcing the parameter estimates to represent the intercepts and slopes of an average-age child in the study. All analyses were run using SPSS version 15.0 for Windows.

\section{RESULTS}

\section{Univariate analyses}

Table 1 presents participants' mean CAFAS scores and standard deviations at waves one through seven, mean difference and standard deviation values from intake to each subsequent wave, mean difference and standard deviation values from wave to wave, and the effect size for intake to subsequent wave changes. CAFAS total scores at waves 2 through 7 were significantly lower (improved) than the CAFAS total score at intake, all $\mathrm{p}$ $<.001$. This finding supported hypothesis (c). The effect sizes for these changes were all large, according to criteria specified by Cohen (1988). Participants improved significantly from wave to wave in succession up to 12 months after intake, all $\mathrm{p}<.05$; after that, scores did not improve significantly between waves. 
TABLE 1. Child and Adolescent Functional Assessment Scale means, standard deviations, mean differences from intake to subsequent waves, and mean wave to wave differences.

\begin{tabular}{lrlccc}
\hline Wave & $\boldsymbol{M}(\boldsymbol{S D})$ & $\begin{array}{c}\text { Intake to wave } \\
\text { difference } \boldsymbol{M}(\boldsymbol{S D})\end{array}$ & $\begin{array}{c}\text { Wave to wave } \\
\text { difference } \boldsymbol{M}(\boldsymbol{S D})\end{array}$ & $\begin{array}{c}\text { Intake to wave } \\
\text { effect size } \\
\text { (Cohen's } \boldsymbol{d})\end{array}$ \\
\hline Intake & 119.36 & $(26.08)$ & & & \\
4 months & 91.91 & $(27.32)$ & $27.45(32.47)^{* * *}$ & $27.45(32.47) * * *$ & 1.03 \\
8 months & 84.04 & $(32.95)$ & $35.32(39.39)^{* * *}$ & $7.87(25.28)^{* *}$ & 1.19 \\
12 months & 73.40 & $(25.73)$ & $45.96(34.30)^{* * *}$ & $10.64(27.22)^{*}$ & 1.77 \\
16 months & 71.70 & $(25.73)$ & $47.66(36.90)^{* * *}$ & $1.70(23.89)$ & 1.84 \\
20 months & 66.17 & $(25.33)$ & $53.19(33.76)^{* * *}$ & $5.53(22.54)$ & 2.07 \\
24 months & 68.72 & $(30.40)$ & $50.64(38.41)^{* * *}$ & $-2.55(24.36)$ & 1.79 \\
\hline
\end{tabular}

Note: $n=47$ due to missing data points for two cases. Probabilities based on dependent samples $t$ tests. Error rate inflated due to the use of unadjusted $p$-values.

${ }^{* * *} p \leq .001 . * * p \leq .01 .{ }^{*} p<.05$.

\section{Indicator analyses}

The average participant's CAFAS score improved by 47.35 points $(S D=42.17)$ from intake to 24-months, a score well-above the 20 point "clinically significant" criterion suggested by Hodges et al. (2004). In total, $77.6 \%$ of the sample achieved clinically significant change from wave 1 to wave 7 .

\section{Multivariate analyses}

Participants' overall change trajectory, initial status, and difference in change trajectory between the first 12 and last 8 months of the study were estimated using hierarchical linear mixed models. We used maximum likelihood estimation with an unstructured covariance structure and random slopes. We first ran an unconditional growth model, which estimated participants' overall change trajectory with no predictor or control variables. In subsequent models we included control and predictor variables in a theoretically-driven fashion, using goodness-of-fit statistics and changes in the random covariance parameters to determine which model best fit the data.

Based on children's medical charts, available control variables included: age, gender, ethnicity, and living arrangement status. Previous work indicated living arrangement status does not significantly moderate outcome in CPSR (Williams \& Sherr, in press) and therefore was not included in any analyses. Gender and ethnicity did not contribute significantly to the model and were therefore excluded, leaving age as the only control variable. Age significantly impacted participants' initial status, but not their slope of change (Wave x Age interaction term); consequently, we included age as a fixed effect for the intercept only. 
Table 2 presents parameter estimates, standard errors, and confidence intervals for the best fitting and final model. The model was based on 348 observations for 49 participants. Model coefficients represent the value for an average age participant (11.5 years old). The average CAFAS score at intake was 127.58, a figure significantly different from zero, $p<.001$, and reflective of a severely impaired group of youth. Each year of age predicted a significant increase in initial CAFAS score of 3.44 points, $p$ $=.001$. Participants experienced a statistically significant improvement in CAFAS score of 13.73 points per 4 months, $p<.001$. After 16 months of treatment, the average participant's CAFAS total score had dropped significantly by 47.63 points, $p<.001$. Finally, the rate of change worsened significantly, by 12.36 points, $p<.001$, during the last 8 months of the study, as compared to the first 12 months, for an average improvement in CAFAS score of (13.73 - 12.36) 1.37 points per four months during the last three waves of the study. These findings support hypotheses (a) through (c).

TABLE 2. Hierarchical linear mixed model parameter estimates, standard errors, and $95 \%$ confidence intervals for CAFAS outcome data during two years of CPSR.

\begin{tabular}{lrrrr}
\hline & & \multicolumn{2}{c}{$95 \%$ confidence interval } \\
Variable & $B$ & \multicolumn{1}{c}{$S E$} & Lower bound & Upper bound \\
\hline Intercept & & & & \\
$\quad$ Initial status & $127.58^{* * *}$ & 4.53 & 118.61 & 136.55 \\
$\quad$ Age (centered) & $3.44^{* * *}$ & 0.92 & 1.58 & 5.29 \\
$\quad$ 16-month & $-47.63^{* * *}$ & 13.32 & -73.87 & -21.38 \\
Rate of change & & & & -10.82 \\
$\quad$ Wave & $-13.73^{* * *}$ & 1.48 & -16.65 & 17.28 \\
$\quad$ First 12 vs. last 8 mos & $12.36^{* * *}$ & 2.50 & 7.44 & \\
\hline
\end{tabular}

Note: $N=49$; CAFAS $=$ Child and Adolescent Functional Assessment Scale. Pseudo- $R^{2}$ (predicted $\mathrm{x}$ observed values $)=.71$.

$* * * p \leq .001$

In order to get an idea of the total variance accounted for by our model, Singer and Willett (2003) suggest computing a pseudo- $R^{2}$ statistic by examining the correlation between the predicted and observed dependent variable values. The resultant correlation, $r=.84, p<.001$ (two tailed) indicated our model accounted for $71 \%$ of the variance in outcome.

\section{DISCUSSION}

This study provided reliable estimates of the rate, course, and significance of change in children's day-to-day functioning during two years of CPSR at the study site. Findings suggest the CPSR program under study is an efficacious form of treatment for children and youth with SED. Children who participated in CPSR achieved statistically and clinically significant improvements in functioning over 24 months of treatment, with 
the brunt of progress occurring during the first year. The variance accounted for by the final regression model was substantial. As practice realities have so far prevented the implementation of a randomized controlled trial (RCT) of CPSR, this evaluation was a logical next step in empirically testing our CPSR program; the findings provide further support of its effectiveness.

Findings presented here suggest CPSR may be as effective as other more thoroughly evaluated and disseminated forms of treatment for SED, such as wraparound (Burchard, Bruns, \& Burchard, 2002; Burns \& Goldman, 1999; Grundle, 2002) and Multisystemic therapy (MST; Henggeler, Schoenwald, Rowland, \& Cunningham, 2002). Table 3 compares our findings to the mean 18-month CAFAS scores reported in a recent study of wraparound, MST, and wraparound plus MST (Stambaugh et al., 2007). Findings from the current study are comparable at 12 months (and thereafter), falling between the average CAFAS scores for wraparound and MST participants at 18 months post-intake. By comparison, participants in the Stambaugh et al. (2007) study received an average of 15 months of wraparound, 5.5 months of MST, and 10.2 months of wraparound plus MST. Although preliminary, this finding warrants further investigation because CPSR may require less start-up and maintenance costs than MST and may be more compatible with Medicaid reimbursement guidelines than wraparound. These findings are significant given the current push for dissemination of evidence-based treatments for SED (Duchnowski, Kutash, \& Friedman, 2002; Hoagwood, Burns, Kiser, Ringeisen, \& Schoenwald, 2001).

TABLE 3. Comparison of current findings to outcomes from other forms of SED-specific treatment.

\begin{tabular}{lcccc}
\hline Outcome & $\begin{array}{c}\text { CPSR }^{\mathrm{a}} \\
M(S E)\end{array}$ & $\begin{array}{c}\text { Wraparound } \\
M(S E)\end{array}$ & $\begin{array}{c}\mathrm{MST}^{\mathrm{c}} \\
M(S E)\end{array}$ & $\begin{array}{c}\text { Wraparound }+\mathrm{MST}^{\mathrm{d}} \\
M(S E)\end{array}$ \\
\hline $\begin{array}{c}\text { Pre CAFAS } \\
\text { total score }\end{array}$ & $119.4(3.80)$ & $113.6(2.41)$ & $109.3(5.76)$ & $131.3(5.06)$ \\
$\begin{array}{c}\text { Post CAFAS } \\
\text { total score } \\
\text { Mean change }\end{array}$ & $73.4(3.75)$ & $79.4(n r)$ & $61.5(n r)$ & $82.3(n r)$ \\
\hline
\end{tabular}

Note: CAFAS = Child and Adolescent Functional Assessment Scale; $n r=$ not reported.

Comparison data reported in Stambaugh et al. (2007).

${ }^{\text {a }}$ Scores differ from Table 1 due to rounding; post-score is mean 12-month CAFAS; $n=47 .{ }^{\mathrm{b}} n$ $=213$; average length of treatment was 15 months. ${ }^{c} n=54$; average length of treatment was 5.5 months. ${ }^{\mathrm{d}} n=53$; average length of treatment was 10.2 months.

Despite their significant improvement, CPSR participants' mean CAFAS total score after two years of treatment was still in the clinically impaired range (i.e. above the 50 point criterion indicating the need for services beyond traditional outpatient care). This is typical of trials involving youth with SED (Duchnowski, Hall, Kutash, \& Friedman, 1998; Greenbaum et al., 1996; Henggeler et al., 1999; Hodges, et al., 2004; Kazdin, Bass, 
Siegel, \& Thomas, 1998), even amongst empirically supported interventions like wraparound and MST (Stambaugh et al., 2007). Such findings underscore the importance of continued support and services for this vulnerable population and their families throughout the lifespan.

Importantly, participant age had a significantly negative impact on severity of impairment at intake but did not moderate participants' rate of change during treatment, suggesting that youth between the ages of 7 to 17 years benefit similarly from the program. This finding differs from other child treatment outcome literature which shows older youth, especially those with conduct or externalizing behavior problems, tend to respond less favorably to treatment (Dishion \& Patterson, 1992; Ruma, Burke, \& Thompson, 1996). The non-significant difference in rate of change is a positive preliminary finding that should be interpreted cautiously given our small sample size.

Unfortunately, practice realities prevented inclusion of a control group in the study; consequently, we cannot conclude that CPSR caused participants' functioning to improve. However, longitudinal studies exploring the trajectory of change in this population's functioning suggest that youth with SED typically experience a worsening of symptoms and functional impairments over time (Armstrong, Dedrick, \& Greenbaum, 2003; Greenbaum et al., 1996; Wagner, 1995; Zigmond, 2006). Combined with the similar performance of CPSR to other targeted, SED-specific interventions (e.g. Stambaugh et al., 2007) the current findings appear promising.

Because CPSR services are open-ended, with no limits on duration posed by statute, the current findings inform utilization management decisions at the study site. Our results suggest participants in this CPSR program experience rapid improvement during the first year of treatment, followed by minimal change during the subsequent eight months. The obvious conclusion might be to limit services to 16 months. However, such a conclusion may be flawed, if the latter phase of treatment is viewed as a "maintenance" phase which provides necessary, ongoing support to youth with serious emotional and behavioral problems. Moreover, these findings are limited to a single CPSR program at one site. Future research is needed to replicate our findings in a larger cross-section of CPSR participants and providers, and to experimentally evaluate the effect of setting limits on the duration of CPSR services.

Although the findings from this study do not generalize to other CPSR programs in the sense of describing their outcomes, the findings do provide a comparative baseline against which other CPSR program sites might compare their outcomes. If some sites do not achieve outcomes comparable to those presented in this study, it may be that certain agency, programmatic, or organizational factors could be targeted for change at the comparison site as a means of improving client outcomes. Conversely, if another program is shown to have outcomes superior to those documented in this study, research might compare and contrast the programs to determine what factors contribute to enhanced functioning for children in the comparison program.

Findings also provide support, at the practice and policy level, for the use of evidence-based cognitive and behavioral interventions for youth with SED. At the practice level, practitioners are guided toward treatment choices that favor here-and-now 
focused skill building, behavior modification, cognitive interventions, and an ecologically valid, coordinated, collaborative, multi-system approach. For policy-makers seeking to increase system efficiency and accountability, these findings offer support for community-based, multi-system, coordinated, cognitive and behavioral interventions with child and adolescent populations. Based solidly in the medical model, third-party payers have been slow to reimburse newer forms of community-based treatment such as CPSR; the current findings offer another round of empirical support for this new wave of services.

This study was not without methodological limitations related to the conduct of research in a community practice setting. First, findings from this study cannot be generalized to other CPSR programs as we employed a small sample from a single CPSR program. As noted above, CPSR programs vary considerably on many important agencylevel variables that may impact client outcomes. Second, our retrospective design and reliance on existing medical records prevented us from examining the impact of important covariates such as the level of family engagement, family functioning, and/ or organizational factors, such as the quality of CPSR Specialist supervision, worker experience level, or other program quality indicators. These variables represent important potential sources of variation in CPSR outcome and should be addressed in a planned, prospective longitudinal study. Third, the study was not controlled or randomized, preventing causal inferences. Fourth, outcome ratings included information from intervention agents, introducing the possibility of rater bias. Finally, due to our retrospective design, we lacked standardized measures of intervention fidelity; this introduced unknown variability into the independent variable.

Perhaps the most significant implication of these findings is they warrant state or federal funding to engage in a large-scale demonstration study of CPSR using an experimental design with random assignment to treatment and control groups. Such a study should include longitudinal analysis of outcomes across multiple domains, including clinical, functional, and systems-level measures (see Hoagwood, Jensen, Petti, \& Burns, 1996, for a comprehensive outcomes model). Future studies should also attempt to tease apart the relative impact of CPSR versus other forms of treatment such as psychotherapy, service coordination, and psychotropic medications, as these often occur concurrently in the clinic setting. Studies are in the planning stages to address these important issues in CPSR.

\section{References}

American Psychiatric Association. (2000). Diagnostic and statistical manual of mental disorders ( $4^{\text {th }}$ ed., text revision). Washington, DC: Author.

Armstrong, K. H., Dedrick, R. F., \& Greenbaum, P. E. (2003). Factors associated with community adjustment of young adults with serious emotional disturbance: A longitudinal analysis. Journal of Emotional and Behavioral Disorders, 11, 66-76.

Bates, M. P. (2001). The Child and Adolescent Functional Assessment Scale (CAFAS): Review and current status. Clinical Child and Family Psychology Review, 4, 63-84. 
Bereiter, C. (1963). Some persisting dilemmas in the measurement of change. In C. W. Harris (Ed.), Problems in the measurement of change (pp. 3-20). Madison, WI: University of Wisconsin Press.

Burchard, J. D., Bruns, E. J., \& Burchard, S. N. (2002). The wraparound approach. In B. J. Burns \& K. Hoagwood (Eds.), Community treatment for youth: Evidence-based interventions for severe emotional and behavioral disorders (pp. 69-90). New York: Oxford University Press.

Burns, B. J., \& Goldman, S. K. (Eds.). (1999). Systems of care: Promising practices in children's mental health, 1998 series: Vol. IV. Promising practices in wraparound for children with serious emotional disturbance and their families. Washington, DC: Center for Effective Collaboration and Practice, American Institutes for Research.

Cohen, J. (1988). Statistical power analysis for the behavioral sciences $\left(2^{\text {nd }}\right.$ ed.). Hillsdale, NJ: Erlbaum.

Dishion, T. J., \& Patterson, G. R. (1992). Age effects in parent training outcome. Behavior Therapy, 23, 719-729.

Duchnowski, A., Hall, K., Kutash, K., \& Friedman, R. (1998). The alternatives to residential treatment study. In M. Epstein, K. Kutash, \& A. Duchnowski (Eds.), Outcomes for children and youth with behavioral and emotional disorders and their families: Programs and evaluation best practices (pp. 55-80). Austin, TX: Pro-ed.

Duchnowski, A. J., Kutash, K., \& Friedman R. M. (2002). Community-based interventions in a system of care and outcomes framework. In B. J. Burns \& K. Hoagwood (Eds.), Community treatment for youth: Evidence-based interventions for severe emotional and behavioral disorders (pp. 16-38). New York: Oxford University Press.

Gibbons, R. D., Hedeker, D., \& Davis, J. M. (1987). Regression toward the mean: More on the price of beer and the salaries of priests. Psychoneuroendocrinology, 12(3), 185-192.

Gibbons, R. D., Hedeker, D., Elkin, I., Waternaux, C., Kraemer, H. C., Greenhouse, J. B., et al. (1993). Some conceptual and statistical issues in analysis of longitudinal data: Application to the NIMH Treatment of Depression Collaborative Research Program dataset. Archives of General Psychiatry, 50, 739-750.

Greenbaum, P. E., Dedrick, R. F., Friedman, R. M., Kutash, K., Brown, E. C., Larieri, S. P., et al. (1996). National adolescent and child treatment study (NACTS): Outcomes for children with serious emotional and behavioral disturbance. Journal of Emotional and Behavioral Disorders, 4, 130-146.

Grundle, T. J. (2002). Wraparound care. In D. T. Marsh \& M. A. Fristad (Eds.). Handbook of serious emotional disturbance in children and adolescents. New York: John Wiley \& Sons, Inc.

Henggeler, S. W., Rowland, M. D., Randall, J., Ward, D. M., Pickrel, S. G., Cunningham, P. B., et al. (1999). Home-based multisystemic therapy as an alternative to the 
hospitalization of youths in psychiatric crisis: Clinical outcomes. Journal of the American Academy of Child and Adolescent Psychiatry, 38, 1331-1339.

Henggeler, S. W., Schoenwald, S. K., Rowland, M. D., \& Cunningham, P. B. (2002). Serious emotional disturbance in children and adolescents: Multisystemic therapy. New York: Guilford Press.

Hoagwood, K., Burns, B. J., Kiser, L., Ringeisen, H., \& Schoenwald, S. K. (2001). Evidence-based practice in child and adolescent mental health services. Psychiatric Services, 52, 1179-1189.

Hoagwood, K., Jensen, P. S., Petti, T., \& Burns, B. J. (1996). Outcomes of mental health care for children and adolescents: I. A comprehensive conceptual model. Journal of the American Academy of Child and Adolescent Psychiatry, 35, 1055-1063.

Hodges, K. (1994). The Preschool and Early Childhood Functional Assessment Scale. Ypsilanti, MI: Eastern Michigan University.

Hodges, K. (2000a). The Child and Adolescent Functional Assessment Scale ( $2^{\text {nd }}$ Rev.). Ypsilanti: Eastern Michigan University.

Hodges, K. (2000b). Child and Adolescent Functional Assessment Scale self-training manual ( $2^{\text {nd }}$ Rev.). Ypsilanti: Eastern Michigan University.

Hodges, K., Doucette-Gates, A., \& Liao, Q. (1999). The relationship between the Child and Adolescent Functional Assessment Scale (CAFAS) and indicators of functioning. Journal of Child and Family Studies, 8, 109-122.

Hodges, K., \& Wong, M. M. (1996). Psychometric characteristics of a multidimensional measure to assess impairment: The Child and Adolescent Functional Assessment Scale. Journal of Child and Family Studies, 5, 445-467.

Hodges, K., \& Wong, M. M. (1997). Use of the Child and Adolescent Functional Assessment Scale to predict service utilization and cost. The Journal of Mental Health Administration, 24, 278-290.

Hodges, K., Wong, M. M., \& Latessa, M. (1998). Use of the Child and Adolescent Functional Assessment Scale (CAFAS) as an outcome measure in clinical settings. The Journal of Behavioral Health Services and Research, 25, 325-336.

Hodges, K., Xue, Y., \& Wotring, J. (2004). Use of the CAFAS to evaluate outcomes for youths with severe emotional disturbance served by public mental health. Journal of Child and Family Studies, 13, 325-339.

Idaho Department of Health and Welfare. (2007). Unpublished raw data obtained through public records request.

Kazdin, A. E. (2003). Research design in clinical psychology (4 ${ }^{\text {th }}$ ed.). Boston, MA: Allyn \& Bacon. 
Kazdin, A. E., Bass, D., Siegel, T., \& Thomas, C. (1998). Cognitive-behavioral therapy and relationship therapy in the treatment of children referred for antisocial behavior. Journal of Consulting and Clinical Psychology, 57, 522-535.

Linn, R. L., \& Slinde, J. A. (1977). The determination of the significance of change between pre- and posttesting periods. Review of Educational Research, 47, 121-150.

Piacentini, J., Bergman, R. L., Jacobs, C., McCracken, J. T., \& Kretchman, J. (2002). Open trial of cognitive-behavior therapy for childhood obsessive-compulsive disorder. Anxiety Disorders, 16, 207-219.

Ringeisen, H., \& Hoagwood, K. (2002). Clinical and research directions for the treatment and delivery of children's mental health services. In D. T. Marsh \& M. A. Fristad (Eds.), Handbook of serious emotional disturbance in children and adolescents (pp. 33-55). New York: John Wiley \& Sons, Inc.

Rogosa, D. R., Brandt, D., \& Zimowski, M. (1982). A growth curve approach to the measurement of change. Psychological Bulletin, 90, 726-748.

Ruma, P. R., Burke, R. V., \& Thompson, R. W. (1996). Group parent training: Is it effective for children of all ages? Behavior Therapy, 27, 159-169.

Singer, J. D., \& Willett, J. B. (2003). Applied longitudinal data analysis: Modeling change and event occurrence. Oxford University Press: New York.

Stambaugh, L. F., Mustillo, S. A., Burns, B. J., Stephens, R. L., Baxter, B., Edwards, D., et al. (2007). Outcomes from wraparound and multisystemic therapy in a center for mental health services system-of-care demonstration site. Journal of Emotional and Behavioral Disorders, 15, 143-155.

Stroul, B., \& Friedman, R. (1986). A system of care for children and youth with severe emotional disturbances (Revised Edition). Washington, DC: Georgetown University Child Development Center, National Technical Assistance Center for Children’s Mental Health.

U. S. Census Bureau. (2008). State and County QuickFacts. Retrieved May 25, 2008, from http://quickfacts.census.gov/qfd/states/16/16027.html.

Vernberg, E. M, Jacobs, A. K., Nyre, J. E., Puddy, R. W., \& Roberts, M. C. (2004). Innovative treatment for children with serious emotional disturbance: Preliminary outcomes for a school-based intensive mental health program. Journal of Clinical Child and Adolescent Psychology, 33, 359-365.

Wagner, M. M. (1995). Outcomes for youths with serious emotional disturbance in secondary school and early adulthood. The Future of Children, 5, 90-112.

Willett, J. B. (1989). Some results on reliability for the longitudinal measurement of change: Implications for the design of studies of individual growth. Educational and Psychological Measurement, 49, 587-602. 
Williams, N. J. (in press). Preliminary evaluation of Children's Psychosocial Rehabilitation for youth with serious emotional disturbance. Research on Social Work Practice.

Williams, N. J., \& Sherr, M. E. (in press). Children's Psychosocial Rehabilitation: Clinical outcomes for youth with serious emotional disturbance living in foster care. Child and Adolescent Social Work Journal.

Zigmond, N. (2006). Twenty-four months after high school: Paths taken by youth diagnosed with severe emotional and behavioral disorders. Journal of Emotional and Behavioral Disorders, 14, 99-108.

\section{Author's note:}

Address correspondence to: Nathanial J. Williams, LCSW, Children's Psychosocial Rehabilitation Program Director, CenterPointe, Inc., 915 Park Centre Way, Suite 7, Nampa, Idaho 83651. E-mail: nwilliams@centerpointeinc.com. 